\title{
52 2013
}

\section{ANIMAL GENETIC RESOURCES}

\section{RESSOURCES}

GÉNÉTIQUES ANIMALES

un journal international

\section{RECURSOS GENÉTICOS ANIMALES}

una revista internacional

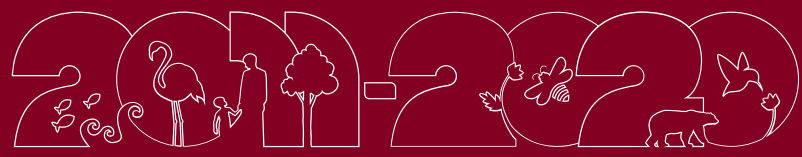

United Nations Decade on Biodiversity

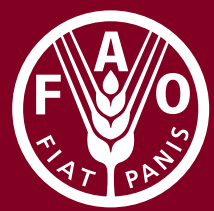


The designations employed and the presentation of material in this information product do not imply the expression of any opinion whatsoever on the part of the Food and Agriculture Organization of the United Nations (FAO) concerning the legal or development status of any country, territory, city or area or of its authorities, or concerning the delimitation of its frontiers or boundaries. The mention of specific companies or products of manufacturers, whether or not these have been patented, does not imply that these have been endorsed or recommended by FAO in preference to others of a similar nature that are not mentioned.

The views expressed in this information product are those of the author(s) and do not necessarily reflect the views or policies of FAO.

Les appellations employées dans ce produit d'information et la présentation des données qui y figurent n'impliquent de la part de l'Organisation des Nations Unies pour l'alimentation et l'agriculture (FAO) aucune prise de position quant au statut juridique ou au stade de développement des pays, territoires, villes ou zones ou de leurs autorités, ni quant au tracé de leurs frontières ou limites. La mention de sociétés déterminées ou de produits de fabricants, qu'ils soient ou non brevetés, n'entraîne, de la part de la FAO, aucune approbation ou recommandation desdits produits de préférence à d'autres de nature analogue qui ne sont pas cités.

Les opinions exprimées dans ce produit d'information sont celles du/des auteur(s) et ne reflètent pas nécessairement les vues ou les politiques de la FAO.

Las denominaciones empleadas en este producto informativo y la forma en que aparecen presentados los datos que contiene no implican, por parte de la Organización de las Naciones Unidas para la Alimentación y la Agricultura (FAO), juicio alguno sobre la condición jurídica o nivel de desarrollo de países, territorios, ciudades o zonas, o de sus autoridades, ni respecto de la delimitación de sus fronteras o límites. La mención de empresas o productos de fabricantes en particular, estén o no patentados, no implica que la FAO los apruebe o recomiende de preferencia a otros de naturaleza similar que no se mencionan.

Las opiniones expresadas en este producto informativo son las de su(s) autor(es), y no reflejan necesariamente los puntos de vista o políticas de la FAO.

FAO encourages the use, reproduction and dissemination of material in this information product. Except where otherwise indicated, material may be copied, downloaded and printed for private study, research and teaching purposes, or for use in non-commercial products or services, provided that appropriate acknowledgement of FAO as the source and copyright holder is given and that FAO's endorsement of users' views, products or services is not implied in any way.

All requests for translation and adaptation rights, and for resale and other commercial use rights should be made via www.fao.org/contact-us/licencerequest or addressed to copyright@fao.org. FAO information products are available on the FAO website (www.fao.org/publications) and can be purchased through publications-sales@ fao.org.

La FAO encourage l'utilisation, la reproduction et la diffusion des informations figurant dans ce produit d'information. Sauf indication contraire, le contenu peut être copié, téléchargé et imprimé aux fins d'étude privée, de recherches ou d'enseignement, ainsi que pour utilisation dans des produits ou services non commerciaux, sous réserve que la FAO soit correctement mentionnée comme source et comme titulaire du droit d'auteur et à condition qu'il ne soit sous-entendu en aucune manière que la FAO approuverait les opinions, produits ou services des utilisateurs.

Toute demande relative aux droits de traduction ou d'adaptation, à la revente ou à d'autres droits d'utilisation commerciale doit être présentée au moyen du formulaire en ligne disponible à www.fao.org/contact-us/licence-request ou adressée par courriel à copyright@fao.org. Les produits d'information de la FAO sont disponibles sur le site web de la FAO (www.fao.org/publications) et peuvent être achetés par courriel adressé à publications-sales@fao.org.

La FAO fomenta el uso, la reproducción y la difusión del material contenido en este producto informativo. Salvo que se indique lo contrario, se podrá copiar, imprimir y descargar el material con fines de estudio privado, investigación y docencia, o para su uso en productos o servicios no comerciales, siempre que se reconozca de forma adecuada a la FAO como la fuente y titular de los derechos de autor y que ello no implique en modo alguno que la FAO aprueba los puntos de vista, productos o servicios de los usuarios.

Todas las solicitudes relativas a la traducción y los derechos de adaptación así como a la reventa y otros derechos de uso comercial deberán dirigirse a www.fao.org/contactus/licence-request o a copyright@fao.org. Los productos de información de la FAO están disponibles en el sitio web de la Organización (www.fao.org/publications) y pueden adquirirse mediante solicitud por correo electrónico a publications-sales@ fao.org.

ISBN 978-92-5-007523-5 (print/version imprimée/edición impresa) E-ISBN 978-92-5-007524-2 (PDF)

(C) FAO 2013
Editor-in-Chief

B. Scherf

Editors

S. Galal; I. Hoffmann

Animal Genetic Resources is an international journal published under the auspices of the Animal Genetic Resources Branch of the Animal Production and Health Division, Food and Agriculture Organization of the United Nations (FAO).

Ressources génétiques animales est un journal international publié sous les auspices de la Sous-Division des ressources génétiques animales de la Division de la production et de la santé animales, Organisation des Nations Unies pour l'alimentation et l'agriculture (FAO).

Recursos genéticos animales es una revista internacional publicada bajo los auspicios de la Subdivisión de los Recursos Genéticos Animales de la División de Producción y Sanidad Animal, la Organización de las Naciones Unidas para la Alimentación y la Agricultura (FAO).

Print edition and institutional subscriptions / Édition imprimée et abonnements pour institutions / Edición de la impresión y suscripciones institucionales: Sales and Marketing Group, Office of Knowledge Exchange, Research and Extension, FAO, Viale delle Terme di Caracalla, 00153 Rome, Italy; Fax: (39) 065705 3360; E-mail / courrier électronique / correo: PublicationsSales@fao.org or through FAO sales agents / ou auprès des agents de vente des publications de la FAO / o a través de los agentes de venta de la FAO.

Online edition: Cambridge University Press online platform at www.journals.cambridge.org/agr. Please visit the homepage to access the fully searchable text with reference linking and also to submit your paper electronically. The electronic version is also available in the library of the Domestic Animal Information System - DAD-IS at www.fao.org/dad-is.

Édition en ligne: Plateforme virtuelle de "Cambridge University Press» accessible sur www.journals.cambridge. org/agr. Veuillez consulter la page d'accueil pour accéder aux textes qui contiennent des liens de référence et dont tout le contenu peut être recherché; ainsi que pour soumettre vos articles par voie électronique. La version électronique est aussi disponible dans la bibliothèque du Système d'information sur la diversité des animaux domestiques, DAD-IS accessible sur www.fao.org/dad-is.

Edición en línea: Plataforma en línea de Cambridge University Press (www.journals.cambridge.org/agr). Por favor, visite la página inicial para acceder a la publicación, en la que pueden llevarse a cabo búsquedas textuales y se proporcionan enlaces a las referencias, y también para someter sus artículos electrónicamente. La versión electrónica está también disponible en la biblioteca del Sistema de Información sobre la diversidad de los animales domésticos, DAD-IS a www.fao.org/dad-is.

Technical enquiries and individual subscriptions / Renseignements techniques et abonnements individuels / Consultas técnicas y suscripciones individuales: Editor-in-Chief, Animal Genetic Resources Branch, FAO, Viale delle Terme di Caracalla, 00153 Rome, Italy; Fax: (39) 065705 5749; E-mail / courrier électronique / correo: AnGR-Journal@fao.org

Submission of manuscripts / Soumission des manuscrits / Envío de los manuscritos electronically via / électroniquement à travers / electrónicamente a través de: http://journals.cambridge.org/action/manuscriptSubmission? jid=AGR

Instructions for contributors / Instructions aux collaborateurs / Instrucciones para los colaboradores see / voir/ ver: http://journals.cambridge.org/action/displayMorelnfo? $\mathrm{jid}=\mathrm{AGR} \&$ type $=$ ifc 